\title{
Synthesis and Antimicrobial Activities of Some Novel Quinoxalinone Derivatives
}

\author{
M.M. Ali ${ }^{1}$, M.M.F. Ismail ${ }^{2}$, M.S.A. El-Gaby ${ }^{3}$, M. A. Zahran $^{1}$ and Y.A. Ammar ${ }^{1} *$ \\ ${ }^{1}$ Department of Chemistry, Faculty of Science, Al-Azhar University, Nasr-City 11884, Cairo, Egypt \\ Fax: 002(02)2629356, E-mail: yossry@yahoo.com \\ ${ }^{2}$ Department of Pharmaceutical Chemistry, Faculty of Pharmacy (Girl's), Al-Azhar University, \\ Nasr-City, Cairo, Egypt \\ ${ }^{3}$ Department of Chemistry, Faculty of Science, Al-Azhar University at Assiut, Assiut 71524, Egypt \\ Fax: 002(088)325436, E-mail: m_elgaby@hotmail.com
}

Received: 29 April 2000 / Accepted: 13 June 2000 / Published: 18 June 2000

\begin{abstract}
Condensation of 4-benzoyl-1,2-phenylenediamine with sodium pyruvate in acetic acid furnished two products which were identified as 6-benzoyl and 7-benzoyl-3-methyl$2(1 \mathrm{H})$ quinoxalinones $(\mathbf{1 a}, \mathbf{b})$. Fusion of $\mathbf{1 a}$ with aromatic aldehydes furnished the styryl derivatives 2a-c. Alkylation of $\mathbf{1 a}, \mathbf{b}$ with dimethyl sulphate or ethyl chloroacetate produced the $\mathrm{N}$-alkyl derivatives $\mathbf{3 a}, \mathbf{b}$ and $\mathbf{4 a}, \mathbf{b}$. Hydrazinolysis of the ester derivative $\mathbf{4 a}$ with hydrazine hydrate afforded the hydrazide derivative $\mathbf{5}$ which underwent condensation with aldehydes to give the corresponding hydrazone derivatives $\mathbf{6 a}, \mathbf{b}$. In addition, chlorination of 1a with thionyl chloride afforded the 2-chloro derivative 7 which was subjected to reaction with sodium azide and n-butylamine to yield the corresponding tetrazolo (8) and n-butylamino (9) derivatives, respectively. The structures of the compounds prepared were confirmed by analytical and spectral data. Also, some of the synthesized compounds were screened for antimicrobial activity.
\end{abstract}

Keywords: Quinoxalinones, 4-benzoyl-1,2-phenylenediamine, antimicrobial activity.

(C) 2000 by MDPI (http://www.mdpi.org). Reproduction is permitted for noncommercial purposes. 


\section{Introduction}

Numerous quinoxaline derivatives are important as antibacterial, antifungal, anticancer, antidepressant and antiinflammatory agents [1-3]. From this point of view and in continuation of our interest on the chemistry of quinoxaline [4-9], it was of interest to synthesized some quinoxalinone derivatives with the aim of investigating their antimicrobial properties.

\section{Results and Discussion}

Condensation of 4-benzoyl-1,2-phenylenediamine with sodium pyruvate in acetic acid at room temperature furnished two products which showed analytical and spectral data in good agreement with the 3-methylquinoxalinone structures 1a,b. The reaction proceeds due to the $(-\mathrm{R})$ effect of the benzoyl group which deactivates the p-amino group, thus the m-amino group initiates the reaction to give 6benzoyl-3-methyl-2(1H)quinoxalinone 1a as the main product $(60 \%)$. The second isomer was obtained in 30\% yield and identified as 7-benzoyl-3-methyl-2(1H)quinoxalinone $\mathbf{1 b}$ (Scheme 1). The ${ }^{1} \mathrm{H}$ NMR spectrum of $1 \mathrm{a}$ in DMSO- $\mathrm{d}_{6}$ exhibited signals at $2.6\left(3 \mathrm{H}, \mathrm{s}, \mathrm{CH}_{3}\right), 8.21(1 \mathrm{H}$, broad, $\mathrm{NH}$; cancelled with $\mathrm{D}_{2} \mathrm{O}$ ). Also, the mass spectrum displayed a molecular ion peak at $\mathrm{m} / \mathrm{z} 264$ (70\%). The proposed fragmentation pattern of $\mathbf{1 a}$ is illustrated in Scheme 2.

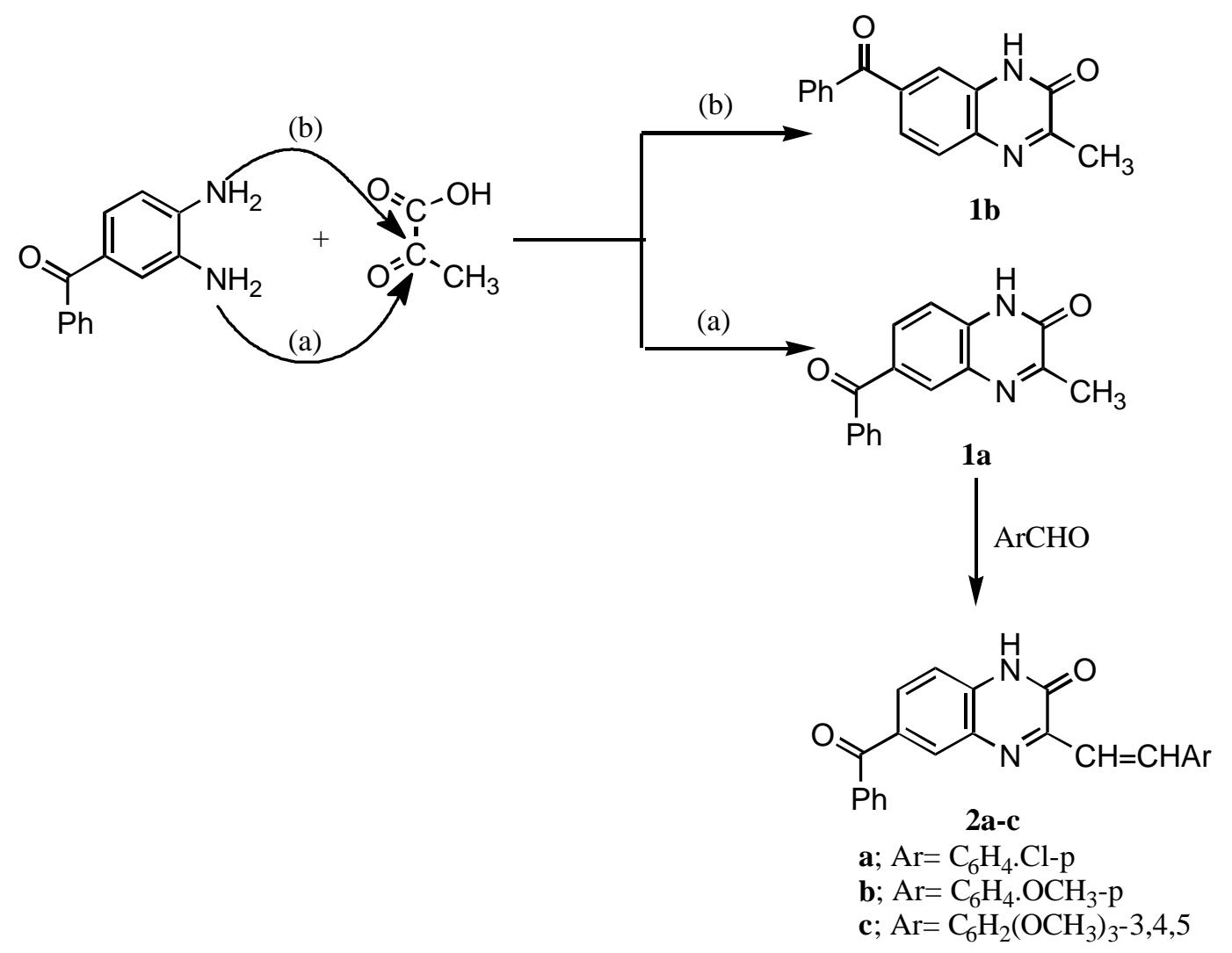

Scheme 1. 


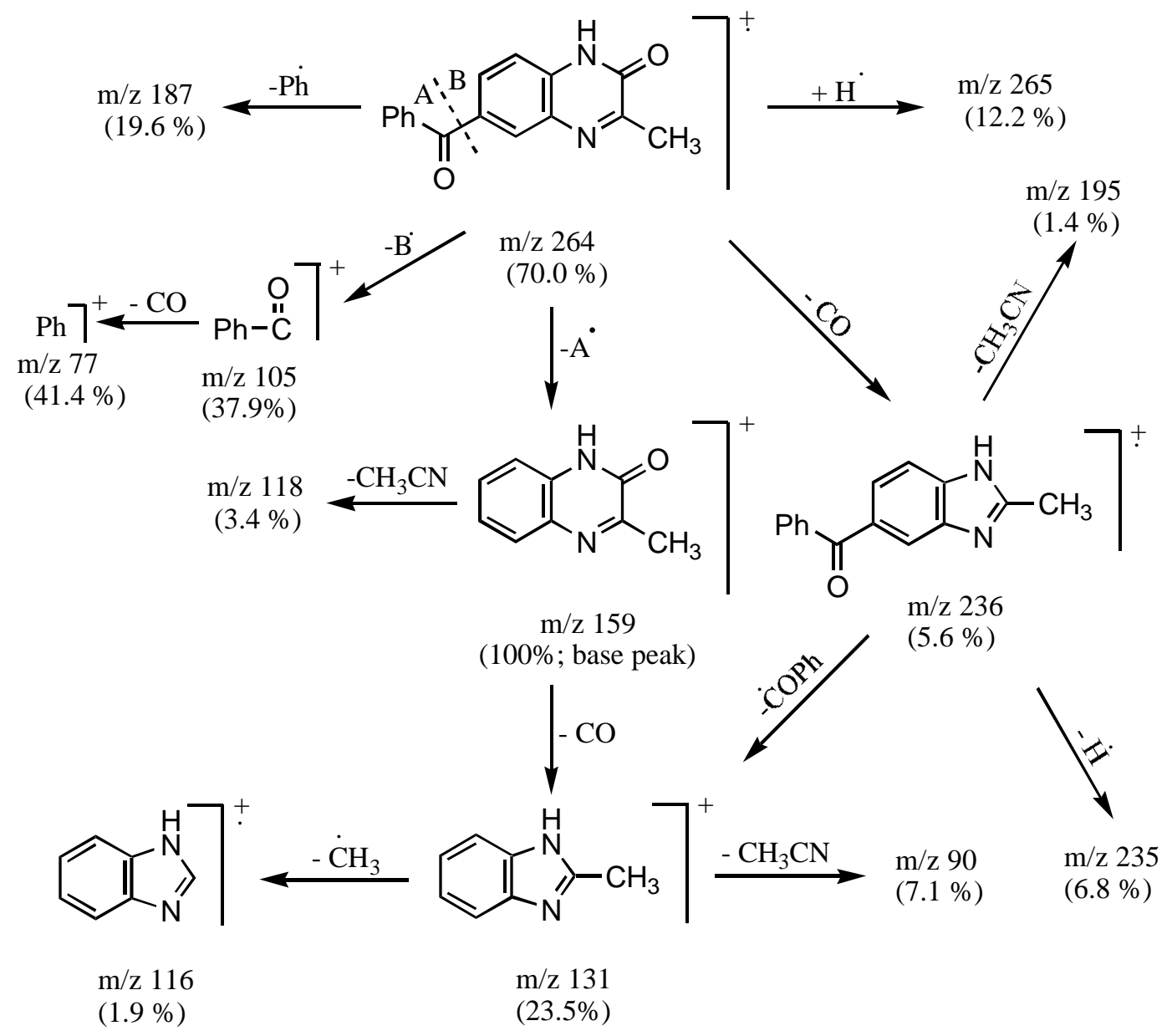

Scheme 2. Fragmentation pattern of compound 1a.

6-Benzoyl-3-substituted styryl-2(1H)quinoxalinones 2a-c were obtained via fusion of 1a with aromatic aldehydes in the presence of piperidine at $160^{\circ} \mathrm{C}$. The ${ }^{1} \mathrm{H}$ NMR of $\mathbf{2 b}$ in DMSO- $\mathrm{d}_{6}$ showed signals at $4.2\left(3 \mathrm{H}, \mathrm{s}, \mathrm{OCH}_{3}\right), 7.20,7.65(2 \mathrm{H}, 2 \mathrm{~s}, \mathrm{CH}=\mathrm{CH})$. The coupling constant of the styryl protons is $14-15$ $\mathrm{Hz}$, indicating trans isomerism at the double bond.

It was reported that quinoxalinone was alkylated using dimethyl sulphate to furnish the corresponding N-methyl derivative [10]. In a similar fashion compounds $\mathbf{1 a}, \mathbf{b}$ were alkylated with dimethyl sulphate in the presence of sodium hydroxide to afford the 6-and 7-benzoyl-1,3-dimethyl$2(1 \mathrm{H})$ quinoxalinones $\mathbf{3 a}, \mathbf{b}$, respectively (Scheme 3). The ${ }^{1} \mathrm{H}$ NMR spectrum of $\mathbf{3 a}$ in $\mathrm{CDCl}_{3}$ showed signals at $2.6\left(3 \mathrm{H}, \mathrm{s}, \mathrm{CH}_{3}\right), 3.6\left(3 \mathrm{H}, \mathrm{s}, \mathrm{N}-\mathrm{CH}_{3}\right)$. Also, when compounds $\mathbf{1 a}$,b were alkylated with ethyl chloroacetate in acetone using potassium carbonate as catalyst, the ester derivatives $\mathbf{4 a}$,b were produced. The ${ }^{1} \mathrm{H}$ NMR spectrum of $\mathbf{4 a}$ in $\mathrm{CDCl}_{3}$ showed 1.4 (3H, t, $\mathrm{CH}_{3}$-ester), $2.4\left(3 \mathrm{H}, \mathrm{s}, \mathrm{CH}_{3}\right), 4.2$ (2H, q, $\mathrm{OCH}_{2}$-ester), 4.9 (2H, s, N-CH$)_{2}$.

Hydrazinolysis of $\mathbf{4 a}$ with hydrazine hydrate afforded 1-[6-benzoyl-3-methyl-2(1H)-quinoxalinone]acetic acid hydrazide (5). Its mass spectrum showed a molecular ion peak at $\mathrm{m} / \mathrm{z} 336\left(\mathrm{M}^{+}, 1.6 \%\right)$ 
and $321\left[\mathrm{M}^{+}-15\left(\mathrm{CH}_{3}.\right)\right]$. Refluxing of the hydrazide derivative 5 with aromatic aldehydes in ethanol furnished the corresponding 1-[6-benzoyl-3-methyl-2(1H)quinoxalinone]acetic hydrazones $6 \mathbf{a}, \mathbf{b}$. The ${ }^{1} \mathrm{H}$ NMR spectrum of $\mathbf{6 b}$ showed signals $2.6\left(3 \mathrm{H}, \mathrm{s}, \mathrm{CH}_{3}\right), 4.0\left(3 \mathrm{H}, \mathrm{s}, \mathrm{OCH}_{3}\right)$ and $9.0(1 \mathrm{H}, \mathrm{s}, \mathrm{N}=\mathrm{CH})$.

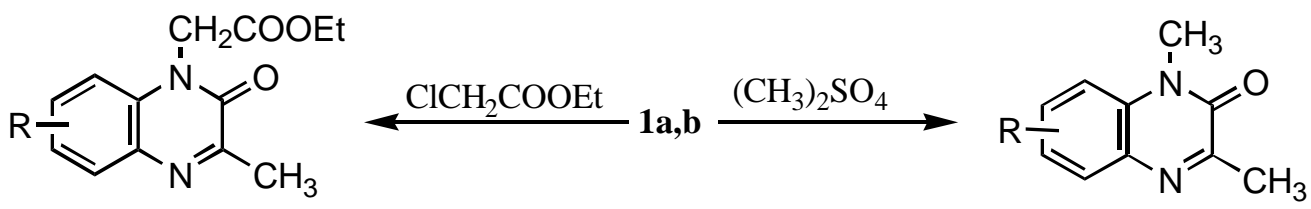
4a, $\mathrm{R}=6$-benzoyl
3a, $\mathrm{R}=6$-benzoyl
b, $\mathrm{R}=7$-benzoyl
b, R= 7-benzoyl

$$
\downarrow \begin{gathered}
4 \mathbf{a} \\
+ \\
\mathrm{NH}_{2} \mathrm{NH}_{2}
\end{gathered}
$$

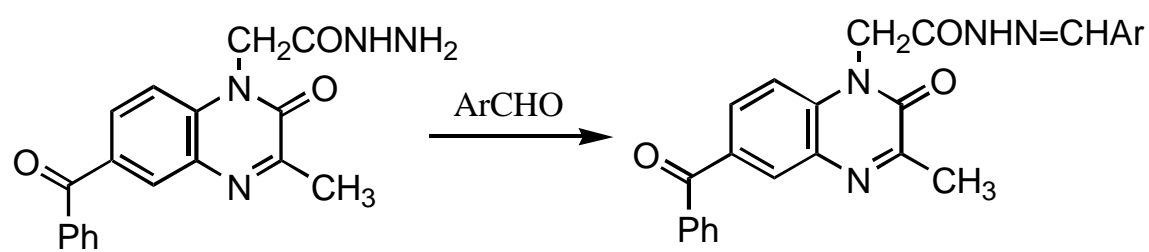

5

6a, $\mathrm{Ar}=\mathrm{C}_{6} \mathrm{H}_{5}$

b, $\mathrm{Ar}=\mathrm{C}_{6} \mathrm{H}_{4} \mathrm{OCH}_{3}-\mathrm{p}$

Scheme 3.

In addition, chlorination of $\mathbf{1 a}$ with thionyl chloride furnished 6-benzoyl-2-chloro-3-methylquinoxaline (7) (Scheme 4).

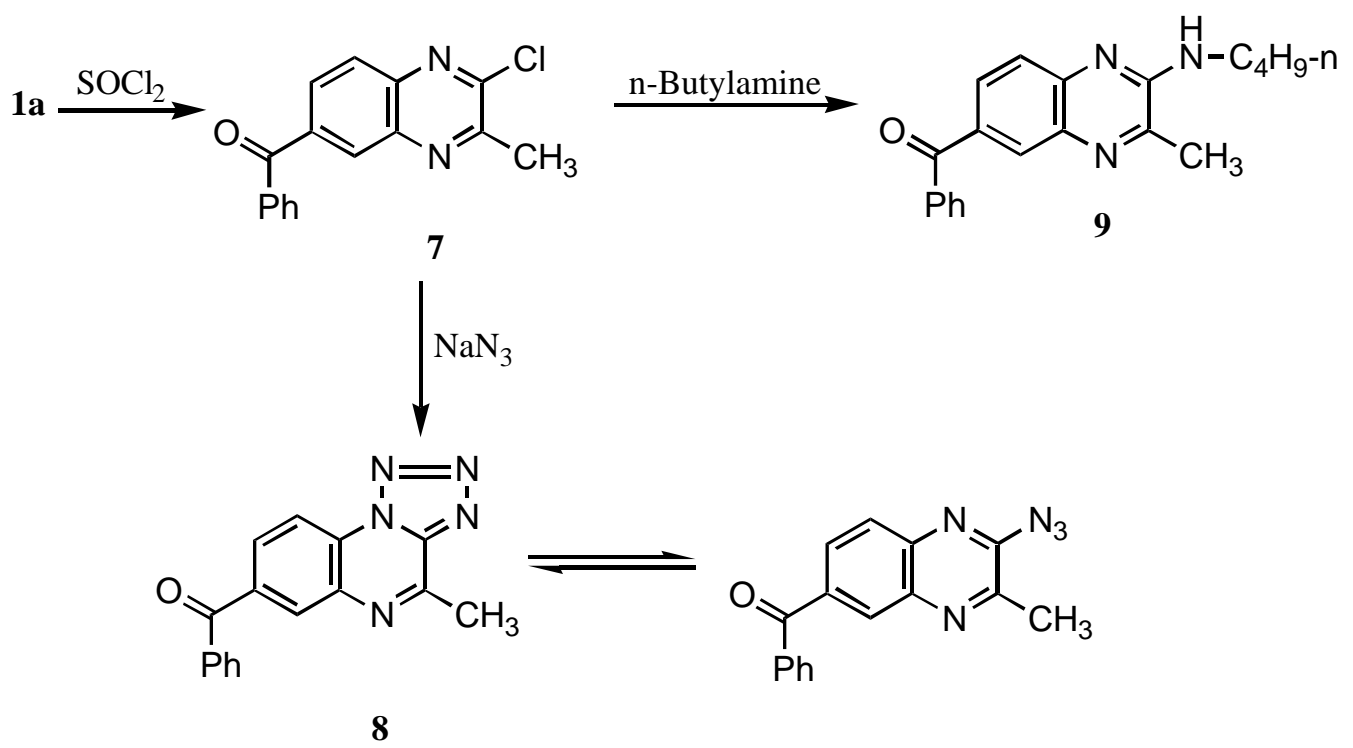

Scheme 4. 
Its IR spectrum revealed the disappearance of the $\mathrm{NH}$ band, present in the parent compound. Also, its mass spectrum showed $\mathrm{m} / \mathrm{z} 267\left[\mathrm{M}^{+}-15\left(\mathrm{CH}_{3}\right), 56.1 \%\right]$. When the chloro derivative 7 was reacted with sodium azide in ethanol, the 7-benzoyl-4-methyltetrazolo[1,5-a]quinoxaline (8) was obtained which was found to be in tautomeric equlibrium with the 2-azido derivative [11]. Its IR spectrum displayed absorption bands at $1700(\mathrm{C}=\mathrm{O})$ and the characteristic absorption band for the azido group at $2240 \mathrm{~cm}^{-1}$ [12]. Also, its mass spectrum displayed a molecular ion peak m/z at $289\left(\mathrm{M}^{+}, 1.6 \%\right)$. Finally, condensation of 7 with n-butylamine in DMF furnished 6-benzoyl-2-butylamino-3-methylquinoxaline (9). Its IR spectrum showed a NH band at $3250 \mathrm{~cm}^{-1}$.

\section{Anitmicrobial activities}

The antimicrobial activities of the synthesized compounds $1 \mathbf{a}, \mathbf{b}, \mathbf{2 b}, \mathbf{4 a}, \mathbf{b}, \mathbf{5}$ and $\mathbf{6} \mathbf{b}$ were determined by the agar diffusion technique [13]. The organisms tested were Staphyloccus aureus (NCTC 7447), Bacillus cereus (ATCC-14579), Serratia marcescens (IMRU 70), Proteus merabitis (NCTC-289), Aspergillus ochraceus Wilhelm (AUCC-230) and Penicillium chrysogenum Thom (AUCC-530). The agar media were inoculated with test organisms and a solution of the tested compound in DMSO (250 $\mu \mathrm{g} / \mathrm{ml})$ was placed separately in cups ( $8 \mathrm{~mm}$ diameter) in the agar medium. Streptomycin $(25 \mu \mathrm{g})$ and mycostatin $(30 \mu \mathrm{g})$ were used as a reference for the antibacterial and antifungal activities, respectively. The inhibition zones were measured after $24 \mathrm{hr}$. incubation. The results of the antimicrobial activity tests are summarized in Table 1.

Table 1. Antimicrobial activity of some synthesized compounds.

\begin{tabular}{|c|l|l|l|l|l|c|}
\hline $\begin{array}{c}\text { Compd. } \\
\text { No. }\end{array}$ & $\begin{array}{l}\text { Staphyloccus } \\
\text { aureus } \\
\text { (NCTC 7447) }\end{array}$ & $\begin{array}{l}\text { Bacillus } \\
\text { cereus } \\
\text { (NCTC 14579) }\end{array}$ & $\begin{array}{l}\text { Serratia } \\
\text { marcesens } \\
\text { (IMRU-70) }\end{array}$ & $\begin{array}{l}\text { Proteus } \\
\text { merabitis } \\
\text { (NCTC-289) }\end{array}$ & $\begin{array}{l}\text { Aspergillus } \\
\text { ochraceus } \\
\text { Wilhelm } \\
\text { (AUCC-230) }\end{array}$ & $\begin{array}{l}\text { Penicillium } \\
\text { chrysogenum } \\
\text { Thom } \\
\text { (AUCC-530) }\end{array}$ \\
\hline $\mathbf{1 a}$ & +++ & ++ & +++ & +++ & ++ & ++ \\
$\mathbf{1 b}$ & ++ & +++ & ++ & ++ & ++ & ++ \\
$\mathbf{2 b}$ & ++ & ++ & +++ & ++ & + & + \\
$\mathbf{4 a}$ & +++ & ++ & ++ & +++ & + & ++ \\
$\mathbf{4 b}$ & +++ & +++ & ++ & ++ & ++ & ++ \\
$\mathbf{5}$ & ++ & ++ & ++ & + & +++ & +++ \\
$\mathbf{6 b}$ & +++ & +++ & +++ & +++ & $\mathrm{R}$ & $\mathrm{R}$ \\
\hline
\end{tabular}

Key: R : Resistant

$+:$ Less active $(0.2-0.5 \mathrm{~mm})$

++ : Moderately active (0.6-1.4 mm)

+++ : Highly active (1.5-2.0 $\mathrm{mm})$ 
Most of the synthesized compounds were found to possess varied antibacterial activities towards all the microorganisms used with minimal inhibitory concentration (MIC). However, none of the tested compounds showed superior activity than the reference drugs.

\section{Experimental}

\section{General}

Melting points were determined by an Electrothermal melting point apparatus and are uncorrected. Elemental analyses were carried out by Microanalytical Unit, Faculty of Science, Cairo University. IR spectra were recorded on a Shimadzu 440 spectrophotometer using $\mathrm{KBr}$ disks. ${ }^{1} \mathrm{H}$ NMR spectra were measured on a Varian EM-360 90 MHz spectrophotometer (Varian, UK) using TMS as internal standard. The mass spectra were run by a Shimadzu-GC-MS-GP 100 EX using the direct inlet system.

\section{3-Methyl-2(1H)quinoxalinones (1a,b)}

A solution of 4-benzoyl-1,2-phenylenediamine $(0.01 \mathrm{~mol})$ in acetic acid $(50 \mathrm{~mL})$ was treated with a solution of sodium pyruvate $(0.01 \mathrm{~mol})$ in a minimum amount of water. The reaction mixture was stirred at room temperature for $2 \mathrm{hr}$ and the solid thus obtained was subjected to fractional crystallization to give products 1a,b (Table 2). Spectral data: 1a: IR $\left(\mathrm{cm}^{-1}\right)$ : $3320(\mathrm{NH}), 2950$ (CH-aliphatic), 1690 $(\mathrm{C}=\mathrm{O}) ;{ }^{1} \mathrm{H}$ NMR (ppm, DMSO-d 6 ): of 1a; $2.47\left(3 \mathrm{H}, \mathrm{s}, \mathrm{CH}_{3}\right), 6.72-7.82(8 \mathrm{H}, \mathrm{m}, \mathrm{Ar}-\mathrm{H}), 8.43(1 \mathrm{H}$, broad, $\mathrm{NH}$; exchangeable with $\left.\mathrm{D}_{2} \mathrm{O}\right)$. Mass spectrum (m/z): $264(\mathrm{M}, 70 \%), 236(5.6 \%), 235(6.8 \%)$, 195 (1.4\%), 187 (19.6\%), 159 (100\%), 131 (23.5\%), 118 (3.4\%), 116 (1.9\%), 105 (37.3\%) and 77 (41.4\%). 1b: ${ }^{1} \mathrm{H}$ NMR (ppm, DMSO-d 6 ): $2.6\left(3 \mathrm{H}, \mathrm{s}, \mathrm{CH}_{3}\right), 8.21(1 \mathrm{H}$, broad, $\mathrm{NH}), 6.8-7.4(8 \mathrm{H}, \mathrm{m}, \mathrm{Ar}-$ $\mathrm{H})$.

\section{6-Benzoyl-3-substituted styryl-2(1H)quinoxalines (2a-c)}

A mixture of $(\mathbf{1 a}, 0.01 \mathrm{~mol})$, the requisite aldehyde $(0.01 \mathrm{~mol})$ and piperidine $(0.5 \mathrm{~mL})$ was fused at $160^{\circ} \mathrm{C}$ for $2 \mathrm{hr}$. The obtained product was crystallized from the appropriate solvent (Table 2) to afford compounds $\mathbf{2 a - c}$.

\section{6-Benzoyl-3-(4-chlorostyryl)-2(1H)quinoxaline (2a)}

IR $\left(\mathrm{cm}^{-1}\right): 3340(\mathrm{NH}), 1680,1650(\mathrm{C}=\mathrm{O}) .{ }^{1} \mathrm{H}$ NMR spectrum of $\mathbf{2 a}$ in DMSO-d $\mathrm{d}_{6}$ exhibited signals at 7.16. $7.78(2 \mathrm{H}, 2 \mathrm{~s}, \mathrm{CH}=\mathrm{CH}), 7.89-8.20(12 \mathrm{H}, \mathrm{m}, \mathrm{Ar}-\mathrm{H}), 8.72(1 \mathrm{H}$, broad, $\mathrm{NH}$; exchangeable with $\left.\mathrm{D}_{2} \mathrm{O}\right)$. 
6-Benzoyl-3-(4-methoxystryryl)-2-(1H)quinoxaline (2b)

IR $\left(\mathrm{cm}^{-1}\right): 3080(\mathrm{NH}), 1682,1645(\mathrm{C}=\mathrm{O}) .{ }^{1} \mathrm{H}$ NMR spectrum of $\mathbf{2 b}$ in DMSO-d $\mathrm{d}_{6}$ exhibited signals at $4.2\left(3 \mathrm{H}, \mathrm{s}, \mathrm{OCH}_{3}\right), 7.20,7.65(2 \mathrm{H}, 2 \mathrm{~s}, \mathrm{CH}=\mathrm{CH}), 7.90-8.4(12 \mathrm{H}, \mathrm{m}, \mathrm{Ar}-\mathrm{H}), 8.6(1 \mathrm{H}, \mathrm{s}, \mathrm{NH}$; exchangeable with $\mathrm{D}_{2} \mathrm{O}$ ).

\section{6-Benzoyl-3-(3,4,5-trimethoxystryryl)-2-(1H)quinoxaline (2c)}

IR $\left(\mathrm{cm}^{-1}\right): 3320(\mathrm{NH}), 1690,1635(\mathrm{C}=\mathrm{O}) .{ }^{1} \mathrm{H}$ NMR spectrum of $2 \mathrm{c}$ in DMSO-d $\mathrm{d}_{6}$ exhibited signals at $3.91\left(6 \mathrm{H}, \mathrm{s}, 2 \mathrm{OCH}_{3}\right), 4.1\left(3 \mathrm{H}, \mathrm{s}, \mathrm{OCH}_{3}\right), 7.30,7.42(2 \mathrm{H}, 2 \mathrm{~s}, \mathrm{CH}=\mathrm{CH}), 7.82-8.32(10 \mathrm{H}, \mathrm{m}, \mathrm{Ar}-\mathrm{H}), 9.21$ $\left(1 \mathrm{H}, \mathrm{s}, \mathrm{NH}\right.$; exchangeable with $\left.\mathrm{D}_{2} \mathrm{O}\right)$.

\section{6-Benzoyl or 7-benzoyl-1,3-dimethyl-2(1H)quinoxalines $\mathbf{( 3 a , b )}$}

Dimethyl sulphate $(0.022 \mathrm{~mol})$ was added to a solution of $(\mathbf{1 a}$ or $\mathbf{1 b} ; 0.01 \mathrm{~mol})$ in sodium hydroxide solution $(10 \%, 50 \mathrm{~mL})$. The reaction mixture was stirred at $60^{\circ} \mathrm{C}$ for $3 \mathrm{hr}$ and the obtained solid was recrystallized to give (3a,b; Table 2). IR spectrum of 3a revealed bands at 2980 (CH-aliphatic) and 1695, 1644 (CO), also ${ }^{1} \mathrm{H}$ NMR spectrum of $\mathbf{3 a}$ in $\mathrm{CDCl}_{3}$ showed signals at $2.6\left(3 \mathrm{H}, \mathrm{s}, \mathrm{CH}_{3}\right), 3.6(3 \mathrm{H}$, s, N-CH $\left.\mathrm{CH}_{3}\right)$, 7.9-8.5 (8H, m, Ar-H). IR ( $\left.\mathrm{cm}^{-1}\right)$ spectrum of 3b: $2950(\mathrm{CH}$-aliphatic), 1693, $1638(\mathrm{C}=\mathrm{O})$. ${ }^{1} \mathrm{H}$ NMR spectrum of $\mathbf{3 b}$ in $\mathrm{CDCl}_{3}$ showed signals at $2.46\left(3 \mathrm{H}, \mathrm{s}, \mathrm{CH}_{3}\right), 3.71\left(3 \mathrm{H}, \mathrm{s}, \mathrm{N}-\mathrm{CH}_{3}\right), 7.82-8.31$ $(8 \mathrm{H}, \mathrm{m}, \mathrm{Ar}-\mathrm{H})$.

\section{Ethyl [6-or 7-benzoyl-3-methyl-2(1H)quinoxalinon-1-yl]acetate (4a,b)}

A mixture of (1a or $\mathbf{1 b} ; 0.01 \mathrm{~mol})$, ethyl chloroacetate $(0.01 \mathrm{~mol})$ and potassium carbonate $(2 \mathrm{~g})$ in acetone $(50 \mathrm{ml})$ was refluxed for $6 \mathrm{hr}$. The obtained product was crystallized from the proper solvent to furnish (4a,b; Table 2). IR spectrum of 4a: 2982 (CH-aliphatic), 1735, $1680(\mathrm{C}=\mathrm{O}) .{ }^{13} \mathrm{C}$ NMR of $4 \mathbf{a}$ DMSO-d 6 showed $15.3\left(\mathrm{CH}_{3}\right.$-ester $), 21.2\left(\mathrm{CH}_{3}\right), 45.4\left(\mathrm{~N}^{-C_{2}}\right), 58.3\left(\mathrm{OCH}_{2}\right.$-ester $), 119-148.6(13 \mathrm{C}$ $\mathrm{sp}^{2}$ carbon atoms) and 168.2, 170.4, 175.8 (3 C=O groups). IR spectrum of $\mathbf{4 b}$ afforded bands at 2985 (CH-aliphatic) and 1730, $1695(\mathrm{C}=\mathrm{O}) .{ }^{1} \mathrm{H} \mathrm{NMR}$ spectrum of $\mathbf{4 b}$ in $\mathrm{CDCl}_{3}$ showed signals at $1.2(3 \mathrm{H}, \mathrm{t}$, $\mathrm{CH}_{3}$-ester), 2.4 (3H, s, $\left.\mathrm{CH}_{3}\right), 4.2\left(2 \mathrm{H}, \mathrm{q}, \mathrm{OCH}_{2}\right.$-ester), $4.9\left(2 \mathrm{H}, \mathrm{s}, \mathrm{N}-\mathrm{CH}_{2}\right)$ and 7.1-8.0(8H, m, Ar-H).

\section{[6-Benzoyl-3-methyl-2-quinoxalinon-1-yl]acetic acid hydrazide (5)}

To a solution of $(\mathbf{4 a}, 0.01 \mathrm{~mol})$ in ethanol $(50 \mathrm{ml})$, hydrazine hydrate $(0.012 \mathrm{~mol})$ was added. The reaction mixture was refluxed for $10 \mathrm{hr}$ and the obtained product was crystallized from the suitable solvent to yield (5; Table 2). Its IR spectrum showed bands at 3300, $3200\left(\mathrm{NH}, \mathrm{NH}_{2}\right), 1690,1675(\mathrm{C}=\mathrm{O})$. Also its mass spectrum showed m/z 336 (1.6\%), 321 (15.8\%), 305 (84.8\%), 277 (5.6\%), 264 (5.0\%), $249(100 \%), 235(3.1 \%), 216(4.5 \%), 188(2.2 \%), 130(2.3 \%), 116(2.3 \%), 105(52.4 \%)$ and 77 
$(53.1 \%)$.

[6-Benzoyl-3-methyl-2(1H)quinoxalinon-1-yl]acetic acid hydrazones $(\mathbf{6 a}, \mathbf{b})$

A solution of the hydrazide derivative $5(0.01 \mathrm{~mol})$ in ethanol $(30 \mathrm{~mL})$ was treated with benzaldehyde or p-methoxybenzaldehyde $(0.01 \mathrm{~mol})$. The reaction mixture was refluxed for $4 \mathrm{hr}$ and the product that obtained after filtration was crystallized from the proper solvent to afford (6a,b; Table 2). IR spectrum of 6a showed bands at 2990 (CH-aliphatic) and 1690, $1670(\mathrm{C}=\mathrm{O}) .{ }^{1} \mathrm{H}$ NMR spectrum of $\mathbf{6 a}$ in $\mathrm{CDCl}_{3}$ exhibited signals at $2.45\left(3 \mathrm{H}, \mathrm{s}, \mathrm{CH}_{3}\right), 4.83\left(2 \mathrm{H}, \mathrm{s}, \mathrm{N}-\mathrm{CH}_{2}\right), 7.40-8.22(13 \mathrm{H}, \mathrm{m}, \mathrm{Ar}-\mathrm{H}), 8.62(1 \mathrm{H}$, broad, $\mathrm{NH}$; exchangeable with $\left.\mathrm{D}_{2} \mathrm{O}\right)$ and $9.10(1 \mathrm{H}, \mathrm{s}, \mathrm{N}=\mathrm{CH}) .{ }^{1} \mathrm{H}$ NMR spectrum of $\mathbf{6 b}$ in $\mathrm{CDCl}_{3}$ exhibited signals at $2.6\left(3 \mathrm{H}, \mathrm{s}, \mathrm{CH}_{3}\right), 4.0\left(3 \mathrm{H}, \mathrm{s}, \mathrm{OCH}_{3}\right), 4.8\left(2 \mathrm{H}, \mathrm{s}, \mathrm{N}-\mathrm{CH}_{2}\right), 7.2-8.1(12 \mathrm{H}, \mathrm{m}, \mathrm{Ar}-\mathrm{H}), 8.5$ $(1 \mathrm{H}$, broad, $\mathrm{NH})$ and $9.0(1 \mathrm{H}, \mathrm{s}, \mathrm{N}=\mathrm{CH})$.

\section{6-Benzoyl-2-chloro-3-methylquinoxaline (7)}

To a solution of 1a, $0.01 \mathrm{~mol})$ in dioxane $(20 \mathrm{~mL})$ and DMF $(5 \mathrm{~mL})$, thionyl chloride $(10 \mathrm{~mL})$ was added. The reaction mixture was refluxed for $4 \mathrm{hr}$ and the obtained solid was crystallized from the proper solvent to afford compound 7 (Table 2). Its mass spectrum showed $\mathrm{m} / \mathrm{z}$ at $267\left[\mathrm{M}^{+}-15\left(\mathrm{CH}_{3}\right)\right.$; 56.1\%], 254 (8.1\%), 242 (14.3\%), 240 (10.4\%), 225 (8.8\%), 184 (2.6\%), 162 (100\%), 105 (COPh; $3.6 \%)$ and $77(73.2)$.

\section{7-Benzoyl-4-methyltetrazolo[1,5-a]quinoxaline (8)}

To a solution of $7(0.01 \mathrm{~mol})$ in ethanol $(50 \mathrm{~mL})$, sodium azide $(0.01 \mathrm{~mol})$ was added and the reaction mixture was refluxed for $6 \mathrm{hr}$. The product obtained after filtration was crystallized from a suitable solvent to furnish 8 (Table 2). Its IR spectrum exhibited the following bands: 2943 (CH-aliphatic), 2240 $\left(\mathrm{N}_{3}\right), 1700(\mathrm{C}=\mathrm{O}), 1609(\mathrm{~N}=\mathrm{N}), 1586(\mathrm{C}=\mathrm{N}) .{ }^{1} \mathrm{H}$ NMR spectrum of 8 in $\mathrm{CDCl}_{3}$ exhibited signals at 2.57 $\left(3 \mathrm{H}, \mathrm{s}, \mathrm{CH}_{3}\right), 7.67-8.21(8 \mathrm{H}, \mathrm{m}, \mathrm{Ar}-\mathrm{H})$. Also, its mass spectrum showed a molecular ion peak at $\mathrm{m} / \mathrm{z}$ $289\left(\mathrm{M}^{+}, 1.6 \%\right), 235$ (1.2\%), 185 (12.4\%), 139 (1.5\%), 105 (COPh, 100\%) and 77 (Ph, 77.2\%).

\section{6-Benzoyl-2-(n-butylamino)-3-methylquinoxaline (9)}

A mixture of $7(0.01 \mathrm{~mol})$ and n-butylamine $(0.01 \mathrm{~mol})$ in DMF $(20 \mathrm{~mL})$ was heated under reflux for $3 \mathrm{hr}$. After allowing the reaction mixture to cool to room temperature, it was poured into cooled water $(100 \mathrm{~mL})$. The insoluble material was filtered off and crystallized from the appropriate solvent to give 9 (Table 1). Its IR spectrum revealed bands at $3250(\mathrm{NH}), 2995$ (CH-aliphatic) and $1688(\mathrm{C}=\mathrm{O})$. Mass spectrum of 9 exhibited a molecular ion peak at m/z $319(25 \%)$ together with base peak at m/z 105 $(100 \%)$. 
Table 2. Physical data of the compounds prepared.

\begin{tabular}{|c|c|c|c|c|c|c|c|}
\hline \multirow{2}{*}{$\begin{array}{c}\text { Compd. } \\
\text { No. }\end{array}$} & \multirow{2}{*}{$\begin{array}{l}\text { M.P. } \\
\left({ }^{\circ} \mathrm{C}\right)\end{array}$} & \multirow{2}{*}{$\begin{array}{c}\text { Solvent } \\
\text { Cryst. }\end{array}$} & \multirow{2}{*}{$\begin{array}{c}\text { Yield } \\
(\%)\end{array}$} & \multirow{2}{*}{$\begin{array}{c}\text { Mol. Formula } \\
\text { (M.wt) }\end{array}$} & \multicolumn{3}{|c|}{ Analyses, $\%$} \\
\hline & & & & & $\mathrm{C}$ & $\mathrm{H}$ & $\mathrm{N}$ \\
\hline \multirow[t]{2}{*}{$1 \mathbf{a}$} & \multirow[t]{2}{*}{$148-50$} & \multirow[t]{2}{*}{$\mathrm{DMF} / \mathrm{H}_{2} \mathrm{O}$} & \multirow[t]{2}{*}{60} & \multirow{2}{*}{$\begin{array}{c}\mathrm{C}_{16} \mathrm{H}_{12} \mathrm{~N}_{2} \mathrm{O}_{2} \\
(264)\end{array}$} & 72.73 & 4.55 & 10.61 \\
\hline & & & & & 72.80 & 4.40 & 10.70 \\
\hline \multirow[t]{2}{*}{$1 b$} & \multirow[t]{2}{*}{$265-7$} & \multirow[t]{2}{*}{ Ethanol } & \multirow[t]{2}{*}{30} & \multirow{2}{*}{$\begin{array}{c}\mathrm{C}_{16} \mathrm{H}_{12} \mathrm{~N}_{2} \mathrm{O}_{2} \\
(264)\end{array}$} & 72.73 & 4.55 & 10.61 \\
\hline & & & & & 72.60 & 4.50 & 10.60 \\
\hline \multirow[t]{2}{*}{$\mathbf{2 a}$} & \multirow[t]{2}{*}{$178-80$} & \multirow[t]{2}{*}{ Ethanol } & \multirow[t]{2}{*}{72} & $\mathrm{C}_{23} \mathrm{H}_{15} \mathrm{~N}_{2} \mathrm{O}_{2} \mathrm{Cl}$ & 71.41 & 3.88 & 7.25 \\
\hline & & & & $(386.5)$ & 71.50 & 3.90 & 7.10 \\
\hline \multirow[t]{2}{*}{$2 b$} & \multirow[t]{2}{*}{$158-60$} & \multirow[t]{2}{*}{ Ethanol } & \multirow[t]{2}{*}{73} & \multirow{2}{*}{$\begin{array}{c}\mathrm{C}_{24} \mathrm{H}_{18} \mathrm{~N}_{2} \mathrm{O}_{3} \\
(382)\end{array}$} & 75.39 & 4.71 & 7.33 \\
\hline & & & & & 75.30 & 4.80 & 7.20 \\
\hline \multirow[t]{2}{*}{$2 \mathrm{c}$} & \multirow[t]{2}{*}{$220-1$} & \multirow[t]{2}{*}{$\mathrm{DMF} / \mathrm{H}_{2} \mathrm{O}$} & \multirow[t]{2}{*}{60} & $\mathrm{C}_{26} \mathrm{H}_{22} \mathrm{~N}_{2} \mathrm{O}_{3}$ & 70.59 & 4.98 & 6.33 \\
\hline & & & & $(442)$ & 70.60 & 5.00 & 6.10 \\
\hline $3 \mathbf{a}$ & $158-60$ & Ethanol & 64 & $\mathrm{C}_{17} \mathrm{H}_{14} \mathrm{~N}_{2} \mathrm{O}_{2}$ & 73.38 & 5.04 & 10.07 \\
\hline & & & & $(278)$ & 73.40 & 5.00 & 10.10 \\
\hline $3 \mathbf{b}$ & $134-6$ & Ethanol & 58 & $\mathrm{C}_{17} \mathrm{H}_{14} \mathrm{~N}_{2} \mathrm{O}_{2}$ & 73.38 & 5.04 & 10.07 \\
\hline & & & & $(278)$ & 73.30 & 5.20 & 10.10 \\
\hline $\mathbf{4 a}$ & $155-7$ & Ethanol & 65 & $\mathrm{C}_{20} \mathrm{H}_{18} \mathrm{~N}_{2} \mathrm{O}_{4}$ & 68.57 & 5.14 & 8.00 \\
\hline & & & & $(350)$ & 68.60 & 5.20 & 8.10 \\
\hline $4 b$ & $180-2$ & Ethanol & 62 & $\mathrm{C}_{20} \mathrm{H}_{18} \mathrm{~N}_{2} \mathrm{O}_{4}$ & 68.57 & 5.14 & 8.00 \\
\hline & & & & (350) & 68.40 & 5.10 & 8.10 \\
\hline 5 & $278-9$ & Ethanol & 73 & $\mathrm{C}_{18} \mathrm{H}_{16} \mathrm{~N}_{4} \mathrm{O}_{3}$ & 64.29 & 4.76 & 16.67 \\
\hline & & & & (336) & 64.10 & 4.80 & 16.70 \\
\hline $6 \mathbf{a}$ & $194-5$ & $\mathrm{DMF} / \mathrm{H}_{2} \mathrm{O}$ & 71 & $\mathrm{C}_{25} \mathrm{H}_{20} \mathrm{~N}_{4} \mathrm{O}_{3}$ & 70.76 & 4.72 & 13.21 \\
\hline & & & & (424) & 70.80 & 5.00 & 13.20 \\
\hline $\mathbf{6 b}$ & $172-3$ & $\mathrm{DMF} / \mathrm{H}_{2} \mathrm{O}$ & 74 & $\mathrm{C}_{26} \mathrm{H}_{22} \mathrm{~N}_{4} \mathrm{O}_{4}$ & 68.72 & 4.85 & 12.34 \\
\hline & & & & $(454)$ & 68.60 & 4.74 & 12.45 \\
\hline 7 & $135-7$ & Benzene & 78 & $\mathrm{C}_{16} \mathrm{H}_{11} \mathrm{~N}_{2} \mathrm{OCl}$ & 67.96 & 3.89 & 9.91 \\
\hline & & & & $(282.5)$ & 67.80 & 3.90 & 10.00 \\
\hline 8 & $140-2$ & Ethanol & 67 & $\mathrm{C}_{16} \mathrm{H}_{11} \mathrm{~N}_{5} \mathrm{O}$ & 66.44 & 3.81 & 24.22 \\
\hline & & & & (289) & 66.58 & 3.70 & 24.14 \\
\hline 9 & $168-70$ & Ethanol & 60 & $\mathrm{C}_{20} \mathrm{H}_{21} \mathrm{~N}_{3} \mathrm{O}$ & 75.24 & 6.58 & 13.17 \\
\hline & & & & (319) & 75.40 & 6.72 & 13.00 \\
\hline
\end{tabular}




\section{References and Notes}

1. Kaneko, C.; Katagiri, S. Asahi Glass Co. Ltd., Japan Kokai Tokkyo Koho JP 1988, 62, 207, 264 (Chem.Abs., 1988, 109, 231061).

2. Sarges, R.; Howard, H.R.; Browne, R.C.; Label, L.A.; Seymour, P.A. J. Med. Chem. 1990, 33, 2240.

3. Kinashi, H.; Otten, S. L.; Dunkan, J. S.; Hutchinson, C. R. J.Antibiot. 1988, 41, 642.

4. Ammar, Y.A.; Ismail, I.M.;.El-Sharief, A.M.Sh.; Mohamed, Y. A.; Amer, R. M. J. Prakt.Chem. 1988, 330, 821.

5. Ammar, Y.A.; Ismail, I. M.; El-Sharief, A. M. Sh.; Mohamed, Y. A.; Amer, R. M. J. Indian Chem. Soc. 1989, 66, 124.

6. Ammar, Y. A. J. Serb. Chem. Soc. 1990, 55, 9, 515.

7. Ammar, Y.A.; Mohamed, Y.A.; El-Sharief, A.M.Sh.; Zahran, M.A. Egypt J.Chem. 1991, 34, 4, 361.

8. Mohamed, Y. A.; Ammar, Y. A.; El-Sharief, A. M. Sh.; Zahran, M. A. Affinidad 1993, 444, 123.

9. El-Shrief, A. M. Sh.; Ali, M.M.; Ammar, Y.A.; Abd Salam, A. A. Pak. J. Sci. Ind. Res. 1996, 1-4, 5 .

10. Basr, M. Z. A.; Mahgoub, S. A.; Abd El-Latif, F.F.; Fahmy, A.M.; Moustafa, O. S. J. Ind. Chem. Soc. 1990, 67, 216.

11. Stole, R.; Hasuch, F. J. Prakt. Chem. 1933, 136, 9.

12. Khalil, Z. H.; Geies, A. A. Phosphorous, Sulfur and Silicon 1990, 60, 223.

13. Carrod, L. P.; Grady, F. D. Antibiotics and Chemotherapy, 3rd. ed.; Churchill Livingstone: Edinburgh, 1972; p. 477.

Samples Availability: Available from the authors.

(C) 2000 by MDPI (http://www.mdpi.org). Reproduction is permitted for noncommercial purposes. 